\title{
Eficácia de plantas para o controle de nematóides gastrintestinais de pequenos ruminantes: revisão de estudos publicados
}

\author{
NERY, P.S.; DUARTE, E.R.; MARTINS, E.R.* \\ Universidade Federal de Minas Gerais - UFMG Instituto de Ciências Agrárias Caixa Postal 135. Av. Universitária \\ 1000, Bairro Universitário. Montes Claros, MG, CEP 39404-006. *ernane-martins@nca.ufmg.br
}

\begin{abstract}
RESUMO: As helmintoses gastrintestinais constituem um dos principais fatores limitantes para a ovinocaprinocultura em todo o mundo e a saúde dos rebanhos depende de um efetivo controle antiparasitário. A resistência aos anti-helmínticos representa um dos entraves para esse controle e a busca por novas bases tem sido um desafio constante. Autilização da fitoterapia na medicina veterinária constitui um campo promissor de pesquisas. Estudos nesta área necessitam da inserção em um contexto agroecológico, tendo como fator limitante o manejo sustentável dos recursos naturais envolvidos. O presente artigo apresenta uma revisão dos estudos de plantas cientificamente testadas no Brasil e em outros países para o controle das parasitoses gastrintestinais em pequenos ruminantes.
\end{abstract}

Palavras-chave: Anti-helmínticos, plantas medicinais, nematóides gastrintestinais, pequenos ruminantes

\begin{abstract}
Plant efficacy in small ruminant gastrointestinal nematode control: a review of published studies. Gastrointestinal helminthiasis has been one of the main limiting factors to small ruminant breeding around the world and the health of these animals depends on an efficient parasitological control. Resistance to anthelmintics represents one of the barriers to this control and the search for new bases has been a constant challenge. The use of phytotherapy in Veterinary Medicine is a promising research field. Studies in this area require the insertion into an agroecological context, presenting as limitation the sustainable management of the involved natural resources. This paper presents a review of studies on plants scientifically tested in Brazil and other countries for gastrointestinal nematode control concerning small ruminants.
\end{abstract}

Key words: anthelmintics, medicinal plants, gastrointestinal nematodes, small ruminants

A produção de ovinos e caprinos tem sido estimulada no Brasil na tentativa de garantir à população uma fonte de renda, além de fornecer carne e leite, fontes protéicas, que passam a fazer parte da dieta desta população. Entretanto, a infecção por nematóides gastrintestinais tem se apresentado como uma das principais causas de perdas econômicas para os produtores de pequenos ruminantes no Brasil e em outras partes do mundo (Girão et al., 1992; Coop \& Kyriazakis, 2001).

Nos últimos anos a sociedade tem priorizado aspectos ambientais, direcionando muitas pesquisas para a descoberta de novas substâncias bioativas que possam ser empregadas no manejo integrado de doenças, com menos efeitos negativos sobre o meio ambiente (Castro, 1989).

A fitoterapia pode constituir alternativa eficaz no controle de parasitas gastrintestinais. Entretanto, na medicina veterinária, ao contrário do que ocorre na medicina humana, estudos envolvendo produtos fitoterápicos para o controle de doenças ainda são escassos. Muitas plantas são, tradicionalmente, conhecidas como possuidoras de atividade antihelmíntica, necessitando, entretanto, que suas eficácias sejam cientificamente comprovadas (Vieira, 2003).

A validação científica dos fitoterápicos é uma etapa inicial obrigatória para a utilização correta de plantas medicinais ou de seus compostos ativos. Os testes in vitro permitem uma avaliação da existência de propriedades anti-helmínticas nos extratos vegetais, e constituem uma etapa preliminar à caracterização de novos compostos ativos presentes nos vegetais, possibilitando a criação de novas alternativas para o controle das parasitoses (Costa et al., 2002).

Recebido para publicação em 27/03/2008

Aceito para publicação em 14/04/2009 
Para que o tratamento anti-helmíntico seja eficiente nos testes de inibição da eclosão de ovos, deve-se impedir que os mesmos eclodam, liberando as larvas que se tornarão infectantes. Neste caso, o ideal é que se tenha um produto que impeça o prosseguimento da fase de blástula. Segundo a classificação do índice de eficácia proposto pela World Association for the Advancement of Veterinary Parasitology (WAAVP) para parasitas adultos, um produto é considerado efetivo quando promover acima de $90 \%$ de ação anti-helmíntica, moderadamente efetivo quando atuar entre 80 a $90 \%$, pouco efetivo quando a ação for entre 60 e $80 \%$ e não efetivo em níveis abaixo de 60\% (Powers et al., 1982).

As pesquisas com plantas para o controle das verminoses gastrintestinais dos animais devem estar inseridas em um contexto agroecológico. Esta inserção deve buscar o manejo sustentável dos recursos naturais, associados à preservação das espécies vegetais em seus ecossistemas e a produção animal. Muitas são as espécies de plantas medicinais conhecidas tradicionalmente por possuírem atividade anti-helmíntica. Entretanto, a eficácia de cada uma delas deve ser avaliada e confirmada cientificamente, considerando suas disponibilidades regionais e seus possíveis efeitos tóxicos para os animais. O presente artigo apresenta uma revisão dos estudos fitoterápicos no Brasil e em outros países para o controle das helmintoses gastrintestinais em pequenos ruminantes. O resumo com nome popular e científico das plantas testadas para o controle desses helmintos encontra-se na Tabela 1, onde as espécies estão listadas em ordem alfabética.

\section{Eficácia anti-helmíntica de plantas no controle de helmintos de pequenos ruminantes \\ $\mathrm{Na}$ tentativa de contribuir para um controle alternativo efetivo de nematóides gastrintestinais em pequenos ruminantes, vários pesquisadores têm se empenhado em testar plantas usadas na medicina popular avaliando a eficácia e segurança das mesmas.}

Nos EUA, Ketzis et al. (2002) trabalhando com óleo essencial de Chenopodium ambrosioides (0,2 $\mathrm{mL} \mathrm{kg}^{-1}$ de peso corporal) obtiveram eficácia igual ao tiabendazole, promovendo a inviabilização de todas as larvas eclodidas de Haemonchus contortus.

Min et al. (2004) avaliaram o efeito de uma pastagem de Lespedeza cuneata sobre a média de OPG e o total de produção de ovos, em caprinos naturalmente infectados. Houve redução nos dois parâmetros avaliados e a porcentagem de ovos que passaram a larvas de terceiro estágio caiu de 99,0 para 58,2\%. Lange et al. (2006) avaliaram a mesma forragem, sobre infecções de $H$. contortus em ovinos. O grupo tratado teve redução de $98 \%$ no OPG no sétimo dia de tratamento. Terril et al. (2007) observaram que a peletização dessa planta reforça a sua eficácia contra nematóides de caprinos e pode facilitar a ampla utilização desta forrageira em pequenos ruminantes.

No Kênia, Gathuma et al. (2004) observaram $77 \%$ de eficácia in vitro do extrato aquoso a quente (24 $\mathrm{mg} \mathrm{mL}^{-1}$ ) de frutos e folhas de Myrsine africana sobre vários nematóides de ovinos. Entretanto, Ghitiori et al. (2002) não obtiveram redução significativa no OPG (ovos por grama de fezes) em carneiros utilizando folhas e frutos da mesma planta. É bem conhecido que os princípios ativos variam entre as partes vegetais, a localização, idade e fase de desenvolvimento da planta. Além disso, o teste in vivo realizado por Githiori et al. (2002) é por si só mais rigoroso na avaliação da eficácia do extrato.

O extrato aquoso da raiz de Albizia anthelmintica e Hilderbrantia sepalosae apresentou excelente ação anti-helmíntica com eficácias superiores a 90\% (Githiori et al., 2003; Gathuma et al., 2004). Já o extrato aquoso de Jasminum abyssinicum (0,3 $\mathrm{L}_{\text {animal }}{ }^{-1}$ ) reduziu em $69 \%$ o OPG de ovinos (Kiprono et al., 2005).

No Zimbabwe, Kahiya et al. (2003) relataram redução de $34 \%$ no OPG de caprinos infectados artificialmente com larvas de $H$. contortus e alimentados com folhas desidratadas de Acacia karoo que constituiu $40 \%$ da dieta.

Na Nigéria, o D-3-O-methylchiroinositol, isolado do caule de Piliostigma thonningiipromove a paralisia de $60 \%$ das larvas de nematóides (Asuzu et al., 1999). Também nesse país, os extratos aquoso e etanólico de Nauclea latifolia foram eficazes na redução do OPG $(93,8 \%)$ em ovinos e diminuiu a sobrevivência de larvas (Ademola et al., 2007b; Onyeyili et al., 2001). Já Alawa et al. (2003) observaram que o extrato aquoso de Annona senegalensis na concentração $7,1 \mathrm{mg} \mathrm{mL}^{-1}$, reduziu a eclodibilidade dos ovos em $88,5 \%$. O mesmo procedimento foi utilizado para Vernonia amigdalina que foi ineficiente nas concentrações avaliadas.

Também na Nigéria, os extratos etanólico e aquoso de Spondias mombin (Ademola et al., 2005) e Spigelia anthelmia (Ademola et al., 2007a) na concentração de $500 \mathrm{mg} \mathrm{kg}^{-1}$ p.c. foram eficazes na redução do OPG (até 65\%) de ovinos. Em testes in vitro, os extratos etanólico e aquoso de Khaya senegalensis inviabilizaram larvas de 1estágio. Nos testes in vivo, $500 \mathrm{mg} \mathrm{kg}^{-1}$ p.c. reduziram $71,5 \%$ do OPG de $H$. contortus e $72,3 \%$ de Trichostrongylus colubriformis. Para Oesphagostomumsp., Strongyloides sp. e Trichuris sp. observaram $100 \%$ de inibição na mesma concentração (Ademola et al., 2004).

Na África do Sul, Bizimenyera et al. (2006) avaliaram a eficácia de extrato acetônico de folhas, caule e raiz de Peltophorum africanum contra $T$. 
colubriformis e nas concentrações cinco e $25 \mathrm{mg}$ $\mathrm{mL}^{-1}$, ocorreu inibição total da eclosão de ovos e do desenvolvimento das larvas. Na Arábia Saudita, AlQarawi et al. (2001), utilizando extratos de Calotropis procera, obtiveram $49 \%$ de redução do OPG. Nos testes in vitro as diluições 1/5 e 1/10 foram letais após 20 minutos de administração.

No Paquistão, extratos de C. procera, Artemisia brevifolia, Swertia chirata e Butea monosperma apresentaram eficácia em testes in vitro (acima de 60\%). No testes in vivo todas as espécies apresentaram redução no OPG acima $50 \%$ (lqbal et al., 2004; lqbal et al., 2005; lqbal et al., 2006a; lqbal et al., 2006b). A espécie Trachyspermum ammi apresentou bom efeito ovicida in vivo reduzindo o OPG em 78\% (Lateef et al., 2006; Jabbar et al., 2006). A ação in vitro e in vivo de um produto comercial contendo taninos foi avaliada contra $H$. contortus e houve redução dose dependente da eclosão de ovos, redução do OPG e melhora na utilização dos nutrientes (lqbal et al., 2007).

Na Etiópia, o pó da raiz de Halothamnus somalensis na dose $2 \mathrm{~g} \mathrm{~kg}^{-1}$ p.c., apresentou $50 \%$ de redução do OPG (Dawo \& Tibbo, 2005). Já os extratos aquosos e hidroalcóolicos de Croton macrostachyus e Ekebergia capensis, bem como o extrato aquoso de Acacia nilotica induziram completa inibição da eclosão dos ovos de $H$. contortus em concentração igual ou inferior a dois $\mathrm{mg} \mathrm{mL}^{-1}$ (Eguale et al., 2006). Eguale et al. (2007) também observaram inibição completa da eclosão de ovos tratados com extratos aquoso e hidroalcóolico de Coriandrum sativum.

Na Suíça, Hördegen et al. (2003) avaliaram os efeitos da administração de extratos de sete plantas, nim (Azadirachta indica), lírio (Melia azedarach), abacaxi (Ananas comosus), Vernonia anthelmintica, Embelia ribes, Fumaria parviflora e Caesalpinia crista, na redução do OPG e de larvas de $H$. contortus e Trichostrongylus colubriformis. Somente o extrato etílico de F. parviflora, na dose de $183 \mathrm{mg} \mathrm{kg}^{-1}$ p.c., promoveu significativa redução no OPG (100\%) e 78,2 e 88,8\% de redução de adultos de $H$. contortus e T. colubriformis. Utilizando um teste de redução modificado methyl-thiazolyltetrazolium (MTT), Hördegen et al. (2006) testaram a atividade anti-helmíntica de seis das plantas citadas anteriormente e os resultados mostraram redução significativa no desenvolvimento lanval chegando a $93 \%$ de inibição com extrato de $A$. indica.

Heckendorn et al. (2007) avaliaram os efeitos da administração de Cichorium intybus, Lotus corniculatus, Onobrychis viciifolia sobre carneiros infectados artificialmente com $\mathrm{H}$. contortus e Cooperia curticeie todas as forragens apresentaram redução significativa no OPG.

Hounzangbe-Adote etal. (2005b), na França, avaliaram o efeito de Zanthoxylum zanthoxyloides (Fagara) administrada na dose de $4 \mathrm{~g} \mathrm{~kg}^{-1}$ p.c. houve redução de $57,8 \%$ na eliminação de ovos e redução de fertilidade das fêmeas dos helmintos. O numero médio de ovos por útero no grupo controle foi de 573,1 e, no grupo tratado, foi de 384,3 . Avaliando o efeito dessa mesma planta e de Newbouldia laevis, Morinda lucida e Carica papaya sobre três estágios de vida de H. contortus, Hounzangbe-Adote et al. (2005a) observaram que essas quatro plantas possuem propriedades anti-helmínticas, sendo demonstrada a redução da motilidade dos adultos desse verme.

Paolini et al. (2003) administraram extrato de quebracho (Schinopsis sp.) para caprinos e obtiveram $64 \%$ de redução do OPG. Com relação à fecundidade das fêmeas de nematóides, o grupo tratado apresentou uma redução de $57 \%$.

No Reino Unido, Marleyetal. (2003) estudaram a helmintose de ovinos naturalmente infectados manejados em piquetes das seguintes forragens, $C$. intybus, L. corniculatus e Lolium perenne/Trifolium repens. Após 35 dias, nos ovinos que pastaram $L$. corniculatus, foram encontrados, em média, 7,6 helmintos e no $L$. perenne/ T.repensforam encontrados 16,0 .

Athanasiadou et al. (2005), avaliaram o efeito anti-helmíntico direto das forragens, Lotus pedunculatus, Hedysarium coronarium, $O$. viciifolia, $C$. intybus e uma mistura de $L$. perenne/T. repens (controle) contra $T$. colubriformis. e não houve evidência de efeito anti-helmíntico direto dessas forragens sobre os helmintos. Vale ressaltar que 0 teor de metabólitos secundários pode variar de acordo com as condições ambientais e que a susceptibilidade dos helmintos também varia de espécie para espécie.

Os extratos de TC de quebracho, foram utilizados em teste de desenvolvimento larval sobre $H$. contortus, Teladorsagia circumcincta, T. colubriformis e Nematodirus battus. Observou-se efeito sobre a motilidade das larvas de terceiro estágio de todas as espécies testadas, sendo este dose dependente. $\mathrm{O}$ extrato de quebracho utilizado in vivo foi eficiente contra T. colubriformise N. battus (Athanasiadou et al., 2001). no Brasil

Eficácia anti-helmíntica de plantas testadas

Apesar de muitas plantas já terem sido descritas como possuidoras de atividade antihelmíntica, poucas foram avaliadas cientificamente. Em um levantamento realizado por Krychak-Furtado (2006), 106 espécies foram citadas com ação antihelmintica, entretanto menos de $17 \%$ tiveram suas eficácias comprovadas. Das espécies identificadas, apenas $17,9 \%$ eram indicadas para tratamento de nematóides de ruminantes.

Nos estudos de Krychak-Furtado (2006), 35 extratos vegetais foram avaliados in vitro contra 


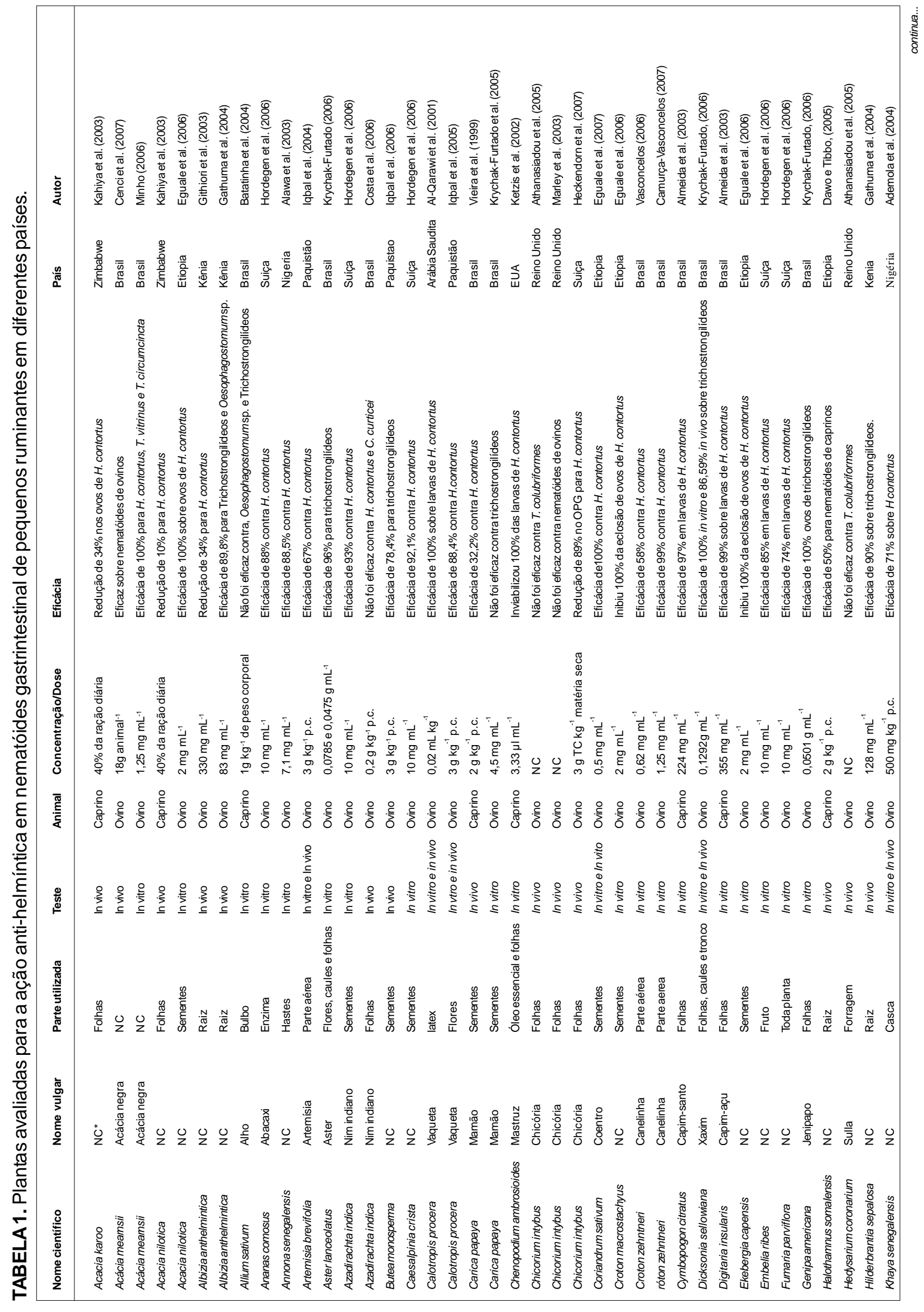

Rev. Bras. PI. Med., Botucatu, v.11, n.3, p.330-338, 2009. 


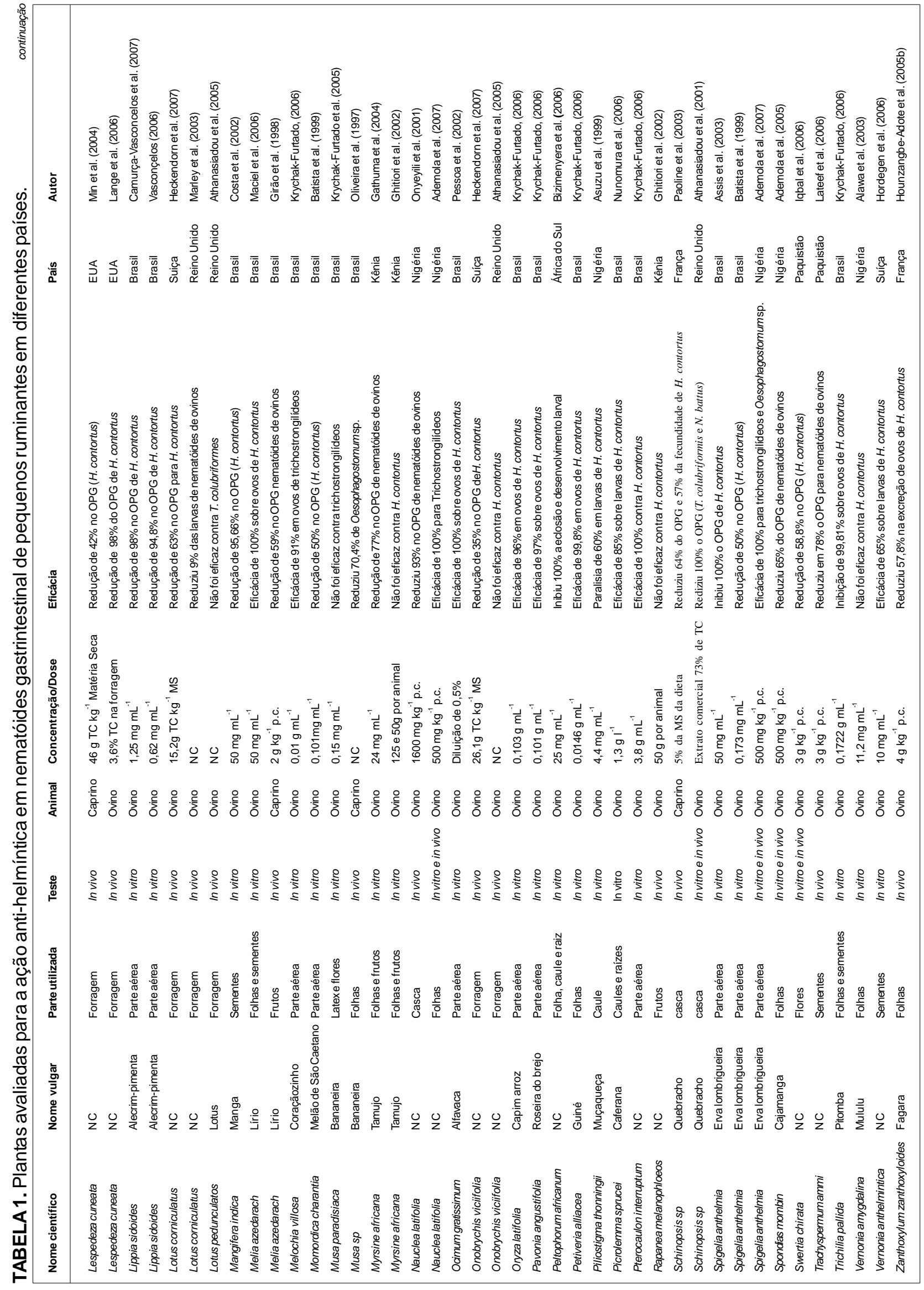

Rev. Bras. PI. Med., Botucatu, v.11, n.3, p.330-338, 2009. 
nematóides gastrintestinais de ovinos. Destes, 13 extratos apresentaram eficiência superior a $80 \%$ : coraçãozinho (Melochia villosa), aster (Aster lanceolatus), capim arroz (Oryza latifólia), roseira do brejo (Pavonia angustifólia), pitomba (Trichilia pallida), guiné (Petiveria alliacea), jenipapo (Genipa americana), xaxim (Dicksonia sellowiana) (pó seco $1 \mathrm{~g}$ ), D. sellowiana (pó seco $2 \mathrm{~g}$ ), D. sellowiana (extrato bruto), $D$. sellowiana (extrato filtrado), Pterocaulon interruptum (fração acetila) e P. interruptum (extrato bruto).

Nos testes in vivo em ovinos, o extrato de $P$. interruptumfoi administrado por via oral, na dosagem de $33,34 \mathrm{mg} \mathrm{kg}^{-1}$ de peso corporal e obteve-se redução de $47 \%$ no número de ovos de trichostrongilídeos eliminados nas fezes. Já a administração de $D$. sellowiana em forma de pó seco, na dose de $5 \mathrm{~g} \mathrm{~kg}^{-1}$ p.c., determinou $86,6 \%$ de redução de ovos dos nematóides gastrintestinais (Krychak-Furtado, 2006).

Melia azedarach, conhecida popularmente como lírio, foi introduzida no Brasil durante a década de 1980 (Maciel et al., 2006). Testes in vivo, utilizando essa planta na dosagem de 2 e 3 gramas de frutos secos e triturados por $\mathrm{kg}$ de p.c., via oral, demonstraram 59 e 54\% de eficácia anti-helmíntica em caprinos, respectivamente. (Girão et al., 1998).

Maciel et al. (2006), testando extratos hexânico, etanólico e clorofórmico de folhas e sementes de M. azedarach sobre H. contortus, observaram $100 \%$ de inibição da eclosão de ovos para o extrato etanólico das folhas nas concentrações de 25 e 50 $\mathrm{mg} \mathrm{mL}^{-1}$. O extrato etanólico das sementes inibiu $100 \%$ da eclosão de ovos em todas as concentrações testadas. O extrato clorofórmico das sementes inibiu $92,4 \%$ da eclosão de ovose $93,5 \%$ do desenvolvimento larval na concentração $50 \mathrm{mg} \mathrm{mL}^{-1}$, enquanto o extrato hexânico não foi eficiente. Os testes fitoquímicos das folhas indicaram a presença de taninos condensados, triterpenóides, esteróides e alcalóides.

Oliveira et al. (1997) observaram redução da infecção por nematóides gastrintestinais em caprinos que receberam, diariamente, folhas de bananeiras (Musa sp.) por um período de 25 dias, quando comparados com o grupo controle. A eficácia da folha de bananeira foi de $57,1 \%$ para Haemonchus sp., $70,4 \%$ para Oesophagostomum sp., $65,4 \%$ para Trichostrongylus sp. e de 59,5\% para Cooperia sp. Por outro lado, Krychak-Furtado et al. (2005) testaram o extrato etanólico e o látex puro de flores de $M$. paradisiaca sobre ovos de nematóides gastrintestinais de ovinos e constataram que nenhum dos tratamentos inibiu o desenvolvimento larval.

Sementes de Carica papaya, trituradas em água e administradas para cabras, reduziram em $32,2 \%$ a contagem de ovos de $H$. contortus, e não foi observada mortalidade de nematóides adultos (Vieira et al., 1999). Krychak-Furtado et al. (2005) testaram o extrato aquoso e o óleo essencial de sementes dessa planta sobre ovos de nematóides de ovinos, porém nenhum dos tratamentos inibiu o desenvolvimento dos ovos desses parasitos.

O extrato aquoso de erva lombrigueira (Spigelia anthelmia) foi testado na dose de $0,17 \mathrm{mg}$ $\mathrm{mL}^{-1}$ sobre a eclosão de ovos de $H$. contortus, obtendo inibição de $50 \%$ dos ovos (Batista et al., 1999). Em outro estudo, as frações com acetato de etila e metanol, na concentração de $50 \mathrm{mg} \mathrm{mL}^{-1}$, inibiram 100 e 97, $4 \%$ da eclosão dos ovos, respectivamente (Assis et al., 2003). Já os extratos, aquoso e etanólico, de caferana (Picrolemma sprucei) inibiram de 85 a $90 \%$ do desenvolvimento de larvas desse nematóide (Nunomura et al., 2006).

Utilizando extrato aquoso de Momordica charantia, Melão de São Caetano, na concentração $0,10 \mathrm{mg} \mathrm{mL}^{-1}$, Batista et al. (1999) observaram $50 \%$ de inibição da eclosão de ovos de $H$. contortus. Posteriormente, Almeida (2005) observou redução média no OPG de $63,1 \%$ em caprinos naturalmente infectados e tratados com folhas dessa planta.

A atividade anti-helmíntica do óleo essencial de alfavaca (Ocimum gratissimum) e do componente, o eugenol nas concentrações 0,5 e $1,0 \%$ apresentaram $100 \%$ de inibição da eclosão dos ovos. O óleo essencial apresentou 14 componentes, sendo encontrado em maior quantidade o eugenol $(43,7 \%)$ e o 1,8-Cineol (32,71\%) (Pessoa et al., 2002).

O extrato hexânico de manga (Mangifera indica) não apresentou efeito ovicida, mas a fração etanólica do extrato hexânico inibiu 95,7\% da eclosão de ovos de $H$. contortus na concentração $50 \mathrm{mg} \mathrm{mL}^{-1}$. Os testes fitoquímicos realizados nesta última fração detectaram proantocianidinas, taninos hidrolisáveis, triterpenos, incluindo saponinas (Costa et al., 2002).

Os estudos in vitro dos extratos aquosos de Cymbopogon citratus (capim-santo) e Digitaria insularis (capim-açu) sobre culturas de larvas de nematóides gastrintestinais de caprinos revelaram uma redução de $97,79 \%$ do número de larvas de $H$. contortus, na concentração de $224 \mathrm{mg} \mathrm{mL}^{-1}$ para o extrato de capimsanto e de $98,94 \%$ para o extrato de capim-açu, na concentração 138,75 mg mL-1 (Almeida et al., 2003). Já o suco de alho (Allium sativum) não foi eficaz sobre o desenvolvimento de ovos e larvas de Strongyloidea em caprinos (Batatinha et al., 2004).

Três plantas citadas como anti-helmínticas Luffa operculata (bucha-paulista) Operculina sp. (batata-de-purga), e Senecio brasiliensis (maria-mole) foram avaliadas in vitro e a batata-de-purga apresentou os melhores resultados na inibição da eclosão de ovos de nematóides (Girão et al., 1998). O farelo de batata-de-purga apresentou redução média de 72,3\% no OPG, 60 dias pós-tratamento de caprinos naturalmente infectados. Animais tratados com semente de jerimum (Cucurbita pepo L.) apresentaram redução média do OPG de 87,3\% (Almeida, 2005). 
O efeito inibitório do óleo essencial de alecrim pimenta (Lippia sidoides) na concentração de $0,02 \mathrm{mg} \mathrm{mL}^{-1}$ foi de $94,84 \% \pm 2,3$, similar ao tiabendazol. A atividade ovicida do óleo essencial de canelinha (Croton zehntneri) e o principal constituinte, o anetol, foram de $58 \%$ e $26,6 \%$ sobre $H$. contortus (Vasconcelos, 2006). Os óleos essenciais de $L$. sidoides, C. zehntnerie os constituintes majoritários reduziram $98 \%$ da eclosão de ovos e inibiram $90 \%$ do desenvolvimento larval do $H$. contortus (CamurçaVasconcelos et al., 2007). O óleo essencial de $L$. sidoides administrado in vivo na concentração de 283 $\mathrm{mg} \mathrm{kg}^{-1}$ apresentou eficácia de 54\%, 14 dias após o tratamento (Camurça-Vasconcelos et al., 2008).

Esta revisão fornece informações relevantes sobre a avaliação da atividade anti-helmíntica de 64 espécies vegetais, das quais 27 apresentaram eficácia acima de 90\%. Em estudos realizados no Brasil, 15 plantas apresentaram eficácia acima de $95 \%$ para inibição do desenvolvimento de trichostrongilídeos. As espécies Aster lanceolatus, Croton zehntneri, Cymbopogon citratus, Dicksonia sellowiana, Digitaria insularis, Genipa americana, Lippia sidoides, Mangifera indica, Melia azedarach, Ocimum gratissimum, Petiveria alliacea, Pterocaulon interruptum, Oryza latifolia, Spigelia anthelmia e Trichilia pallida, que estão adaptadas em diferentes regiões geográficas, foram as que revelaram resultados mais promissores nas pesquisas brasileiras.

Diversos autores em outros paises relatam o potencial promissor de plantas ricas em taninos no controle de nematóides e esse metabólito secundário está presente em muitas plantas nativas do cerrado e da caatinga, indicando um amplo campo de pesquisas de fitoterápicos com ação anti-helmíntica nessas vegetações brasileiras. O conhecimento e o melhor aproveitamento dessas espécies poderão constituir alternativa ecológica, social e economicamente viável.

De forma geral os estudos são realizados sob diferentes abordagens, entretanto, é necessária a complementação dos dados com análises clínicas, toxicológicas e fitoquímicas para a validação de experimentos in vivo. Os dados compilados nesta revisão poderão nortear a elaboração e o registro de novos produtos anti-helmínticos para animais nas entidades competentes. Espera-se que estes dados científicos alcancem o produtor rural, levando consigo os benefícios econômicos, ambientais e de saúde pública, advindos da utilização de métodos de controle anti-helmínticos alternativos aos convencionais.

\section{AGRADECIMENTO}

À Coordenação de Aperfeiçoamento de Pessoal de Nível Superior (CAPES) e ao Banco do Nordeste/FUNDECl.

\section{REFERÊNCIA}

ADEMOLA, I.O.; FAGBEMI, B.O.; IDOWU, S.O. Evaluation of the anthelmintic activity of Khaya senegalensis extract against gastrointestinal nematodes of sheep: in vitro and in vivo studies. Veterinary Parasitology, v.122, p.151-64, 2004.

ADEMOLA, I.O.; FAGBEMI, B.O.; IDOWU, S.O. Anthelmintic activity of extracts of Spondias mombin against gastrointestinal nematodes of sheep: studies in vitro and in vivo. Tropical Animal Health and Production, v.37, n.3, p.223-35, 2005.

ADEMOLA, I.O.; FAGBEMI, B.O.; IDOWU, S.O. Anthelmintic activity of Spigelia anthelmia extract against gastrointestinal nematodes of sheep. Parasitology Research, v.101, p.63-9, 2007a.

ADEMOLA, I.O.; FAGBEMI, B.O.; IDOWU, S.O. Anthelmintic efficacy of Nauclea Latifolia extract against gastrointestinal nematodes of sheep: In Vitro and In Vivo studies. African Journal of Traditional, Complimentary and Alternative Medicines, v.4, n.2, p.148-56, 2007b.

AL-QARAWI, A.A. et al. A preliminary study on the anthelmintic activity of Calotropis procera latex against Haemonchus contortus infection in Najdi sheep. Veterinary Research Communications, v.25, n.1, p.6170, 2001.

ALAWA, C.B.I. et al. In vitro screening of two Nigerian medicinal plants (Vernonia amygdalina and Annona senegalensis) for anthelmintic activity. Veterinary Parasitology, v.113, p.73-81, 2003.

ALMEIDA, M.A.O. et al. Efeitos dos extratos aquosos de folhas de Cymbopogon citratus (DC.) Stapf (Capimsanto) e de Digitaria insularis (L.) Fedde (Capim-açu) sobre cultivos de larvas de nematóides gastrintestinais de caprinos. Revista Brasileira de Parasitologia Veterinária, v.12, n.3, p.125-9, 2003.

ALMEIDA, W.V.F. Uso de plantas medicinais no controle de helmintos gastrintestinais de caprinos naturalmente infectados. 2005. 85p. Dissertação (Mestrado - Área de Concentração em Controle de Parasitismo em Sistemas Agrossilvopastoris) - Centro de Saúde e tecnologia Rural, Universidade Federal de Campina Grande, Patos.

ASSIS, L.M. et al. Ovicidal and larvicidal activity in vitro of Spigelia anthelmia Linn. extracts on Haemonchus contortus. Veterinary Parasitology, v.117, p.43-9, 2003. ASUZU, I.U.; GRAY, A.I.; WATERMAN, P.G. The anthelmintic activity of D-3-O-methylchiroinositol isolated from Piliostigma thonningii stem bark. Fitoterapia, v.70, p.779, 1999.

ATHANASIADOU, S. et al. Direct anthelmintic effects of condensed tannins towards different gastrointestinal nematodes of sheep: in vitro and in vivo studies. Veterinary Parasitology, v.99, p.205-19, 2001.

ATHANASIADOU, S. et al. Testing for direct anthelmintic effects of bioactive forages against Trichostrongylus colubriformis in grazing sheep. Veterinary Parasitology, v.127, p.233-43, 2005.

BATATINHA, M.J.M. et al. Efeitos do suco de alho (Allium sativum Linn.) sobre nematódeos gastrintestinais de caprinos. Ciência Rural, v.34, n.4, p.1265-6, 2004.

BATISTA, L.M. et al. Atividade ovicida e larvicida in vitro de Spigelia anthelmia e Momordica charantia contra o 
nematódeo Haemonchus contortus. Ciência Animal, v.9, p.67-3, 1999.

BIZIMENYERA, E.S. et al. In vitro activity of Peltophorum africanum Sond. (Fabaceae) extracts on the egg hatching and larval development of the parasitic nematode Trichostrongylus colubriformis. Veterinary Parasitology, v.142, p.336-43, 2006.

CAMURÇA-VASCONCELOS, A.L.F. et al. Anthelmintic activity of Croton zehntneri and Lippia sidoides essential oils. Veterinary Parasitology, v.148, p.288-94, 2007.

CAMURÇA-VASCONCELOS, A.L.F. et al. Anthelmintic activity of Lippia sidoides essential oil on sheep gastrointestinal nematodes. Veterinary Parasitology, v.154, p.167-70, 2008.

CASTRO, A.G. Defensivos agrícolas como um fator ecológico. Jaguariúna: EMBRAPA - CNPDA, 1989. 20p. (Documento 6).

CENCl, F.B. et al. Effects of condensed tannin from Acacia mearnsii on sheep infected naturally with gastrointestinal helminthes. Veterinary Parasitology, v.144, p.132-7, 2007.

COOP, R.L.; KYRIAZAKIS, I. Influence of host nutrition on the development and consequences of nematode parasitism in ruminants. Trends in Parasitology, v.17, p.325-30, 2001.

COSTA, C.T.C. et al. Efeito ovicida de extratos de sementes de Mangifera indica I. sobre Haemonchus contortus. Revista Brasileira Parasitologia Veterinária, v.11, n.2, p.57-60, 2002.

COSTA, C.T.C. et al. Anthelmintic activity of Azadirachta indica A. Juss against sheep gastrointestinal nematodes. Veterinary Parasitology, v.137, p.306-10, 2006.

DAWO, F.; TIBBO, M. Anthelmintic effect of Halothamus somalensis in Arsi-Bale goats. Livestock Research for Rural Development, v.17, n.68, 2005. Disponível em <http://www.cipav. org.co//rrd//rrd17/6/dawo17068.htm>. Acesso em: 18 jan. 2008.

EGUALE, T. et al. In vitro anthelmintic activities of four Ethiopian medicinal plants against Haemonchus contortus. Pharmacologyonline, v.3, p.153-65, 2006.

EGUALE, T. et al. In vitro and in vivo anthelmintic activity of crude extracts of Coriandrum sativum against Haemonchus contortus. Journal of Ethnopharmacology, v.110, p.428-33, 2007.

GATHUMA, J.M. et al. Efficacy of Myrsine africana, Albizia anthelmintica and Hilderbrantia sepalosa herbal remedies against mixed natural sheep helminthosis in Samburu district, Kenya. Journal of Ethnopharmacology, v.91, p.7-12, 2004.

GIRÃO, E.S. et al. Ocorrência e distribuição estacional de helmintos gastrintestinais de caprinos no município de Teresina, Piauí. Ciência Rural, v.22, p.197-202, 1992. GIRÃO, E.S. et al. Identificação e Avaliação de Plantas Medicinais com Efeito Anti-Helmíntico em Caprinos. Teresina: EMBRAPA-CPAMN, 1998. 42p. (Documento 29) GITHIORI, J.B. et al. Anthelmintic activity of preparations derived from Myrsine africana and Rapanea melanophloeos against the nematode parasite, Haemonchus contortus, of sheep. Journal of Ethnopharmacology, v.80, p.187-91, 2002.

GITHIORI, J.B. et al. The anthelmintic efficacy of the plant, Albizia anthelmintica, against the nematode parasites $H$. contortus of sheep and Heligmosomoides polygyrus of mice. Veterinary Parasitology, v.116, p.23-34, 2003. HECKENDORN, F. et al. Individual administration of three tanniferous forage plants to lambs artificially infected with Haemonchus contortus and Cooperia curticei. Veterinary Parasitology, v.146, p.123-34, 2007.

HÖRDEGEN, P. et al. The anthelmintic efficacy of five plant products against gastrointestinal trichostrongylids in artificially infected lambs. Veterinary Parasitology, v.17, p.51-60, 2003.

HÖRDEGEN, P. et al. In vitro screening of six anthelmintic plant products against larval Haemonchus contortus with a modified methyl-thiazolyl-tetrazolium reduction assay. Journal of Ethnopharmacology, v.108, p.85-9, 2006.

HOUNZANGBE-ADOTE, M.S. et al. In vitro effects of four tropical plants on three life-cycle stages of the parasitic nematode, Haemonchus contortus. Research in Veterinary Science, v.78, p.155-60, 2005a.

HOUNZANGBE-ADOTE, M.S. et al. In vivo effects of Fagara leaves on sheep infected with gastrointestinal nematodes. Tropical Animal Health and Production, v.37, n.3, p.205-14, 2005b.

IQBAL, Z. et al. Anthelmintic activity of Artemisia brevifolia in sheep. Journal of Ethnopharmacology, v.93, p.265-8, 2004.

IQBAL, Z. et al. Anthelmintic activity of Calotropis procera (Ait.) Ait. F. flowers in sheep. Journal of Ethnopharmacology, v.102, p.256-61, 2005

IQBAL, Z. et al. Anthelmintic activity of Swertia chirata against gastrointestinal nematodes of sheep. Fitoterapia, v.77, p.463-5, 2006a.

IQBAL, Z. et al. In vivo anthelmintic activity of Butea monosperma against Trichostrongylid nematodes in sheep. Fitoterapia, v.77, p.137-40, 2006b.

IQBAL, Z. et al. Direct and indirect anthelmintic effects of condensed tannins in sheep. Veterinary Parasitology, v.144, p.125-31, 2007.

JABBAR, A.; IQBAL, Z.; KHAN, M.N. In vitro anthelmintic activity of Trachyspermum ammiseeds. Pharmacognosy Magazine, v.2, n.6, p.126-9, 2006.

KAHIYA, C.; MUKARATIRWA, S.; THAMSBORG, S.M. Effects of Acacia nilotica and Acacia karoo diets on Haemonchus contortus infection in goats. Veterinary Parasitology, v.115, p.265-74, 2003.

KETZIS, J.K. et al. Chenopodium ambrosioides and its essential oil as treatments for Haemonchus contortus and mixed adult-nematode infections in goats. Small Ruminantes Research, v.44, p.193-200, 2002.

KIPRONO, P.C.; WANJALA, F.M.; KOMEN, C. Efficacy of Jasminum abyssinicum treatment against Hemonchus contortus in sheep. African Journal Traditional, Complementary and Alternative Medicines, v.2, n.3, p.264-8, 2005.

KRYCHAK-FURTADO, S. Alternativas fitoterápicas para o controle da verminose ovina no estado do Paraná: testes in vitro e in vivo. 2006. 147p. Tese (Doutorado em Agronomia) - Universidade Federal do Paraná, Curitiba. KRYCHAK-FURTADO, S. et al. Efeito de Carica papaya I. (caricaceae) e Musa paradisiaca Linn. (musaceae) sobre o desenvolvimento de ovos de nematódeos gastrintestinais de ovinos. Arquivos do Instituto Biológico, v.72, n.2, p.191-7, 2005.

LANGE, K.C. et al. Effect of sericea lespedeza (Lespedeza cuneata) fed as hay, on natural and 
experimental Haemonchus contortus infections in lambs. Veterinary Parasitology, v.141, p.273-8, 2006.

LATEEF, M. et al. Preliminary screening of Trachyspermum ammi (L.) seed for anthelmintic activity in sheep. Tropical Animal Health and Production, v.38, p.491-6, 2006.

MACIEL, M.V. et al. Ovicidal and larvicidal activity of Melia azedarach extracts on Haemonchus contortus. Veterinary Parasitology, v.140, p.98-104, 2006.

MARLEY, C.L. et al. The effect of birdsfoot trefoil (Lotus corniculatus) and chicory (Cichorium intybus) on parasite intensities and performance of lambs naturally infected with helminth parasites. Veterinary Parasitology, v.112, p.147-55, 2003.

MIN, B.R. et al. The effect of short-term consumption of a forage containing condensed tannins on gastro-intestinal nematode parasite infections in grazing wether goats. Small Ruminant Research, v.51, p.279-83, 2004

NUNOMURA, R.C.S. et al. In vitro studies of the anthelmintic activity of Picrolemma sprucei Hook. f. (Simaroubaceae). Acta Amazonica, v.36, n.3, p.327-30, 2006.

OLIVEIRA, D.B. et al. Atividade anti-helmíntica da babaneira (Musa sp) em caprinos. In: CONGRESSO BRASILEIRO DE PARASITOLOGIA, 15., 1997. Salvador, BA. Anais... Salvador: Sociedade Brasileira de Parasitologia, 1997. p.65.

ONYEYILI, P.A. et al. Anthelmintic activity of crude aqueous extract of Nauclea latifolia stem bark against ovine nematodes. Fitoterapia, v.72, p.12-21, 2001.

PAOLINI, V. et al. Effects of condensed tannins on goats experimentally infected with Haemonchus contortus. Veterinary Parasitology, v.113, p.253-61, 2003.

PESSOA, L.M. et al. Anthelmintic activity of essential oil of Ocimum gratissimum Linn. and eugenol against Haemonchus contortus. Veterinary Parasitology, v.109, p.59-63, 2002.

POWERS, K.G. et al. World Association for the Advancement of Veterinary Parasitology (W.A.A.V.P.) guidelines for evaluating the efficacy of anthelmintics in ruminants (bovine and ovine). Veterinary Parasitology, v.10, n.4, p.265-84, 1982.

TERRILL, T.H. et al. Effect of pelleting on efficacy of sericea lespedeza hay as a natural dewormer in goats. Veterinary Parasitology, v.146, p.117-22, 2007.

VASCONCELOS, A.L.C.F. Avaliação da atividade antihelmíntica dos óleos essenciais de Lippia sidoidese croton zehntneri sobre nematóides gastrintestinais de ovinos. 2006. 83p. Tese (Doutorado - Área de Concentração em Reprodução e Sanidade Animal) Faculdade de Medicina Veterinária, Universidade Estadual do Ceará, Fortaleza.

VIEIRA, L.S. et al. Evaluation of anthelmintic efficacy of plants avaivable in Ceará State, Northeast Brazil, for the control of goat gatrointestinal nematodes. Revue Medicine Veterinaire, v.150, n.5, p.447-52, 1999. 